\title{
EL ESPACIO PERDIDO: TÁCTICAS DE CONTRACULTURA EN EL ARTE (2016)
}

\author{
Ventura Alejandro Pérez Suárez \\ Universidad de Vigo. Dpto. Escultura \\ Grupo de investigación Procesos de Resistencia de Arte Contemporáneo Español (PRACE)
}

\section{Resumen}

El presente documento analiza la contracultura y cultura de masas bajo la espacialidad. Esta segunda mantendrá su posicionamiento central absorbiendo subculturas y bloqueando contraculturas por medio de métodos de indefinición como la asimilación, coerción y ocultamiento. En estos procesos se parte de diferentes críticos como G. Cortés, Sennett, Roszak, Comité Invisible y las obras propuestas de Paula Artés y Leendert van der Meer, que trabajan desde el espacio como modelo crítico contracultural. De este modo y por un lado, "L.2/86" de Artés se establecerá como un desmantelamiento de aquella estructura cultural basada en la vigilancia especializada. Por otro lado, "Claiming Space" de van der Meer tomará la videocámara como herramienta legada de una arquitectura de control, para finalmente intervenir y retomar el espacio público de manera subversiva.

Palabras clave: REVELAR; REBELAR; ARQUITECTURA; VIDEOVIGILANCIA; ESPACIALIDAD

\section{THE LOST SPACE: COUNTERCULTURE TACTICS IN THE ART (2016)}

\begin{abstract}
The current document analyzes the counterculture, main culture and their spatiality. The second concept is held in its main position absorbing and blocking subcultures and countercultures by methods of indefiniteness as assimilation, coercion and concealment. So, this processes have been studied by several critics as G. Cortés, Roszak, Invisible committee and the pieces of Paula Artés and Leendert van der Meer, whose works are related to the space as a critic countercultural model. Thereby and on the one hand, "L.2/86" by Artés is the detection of the culture structure based on the specialized surveillance. On the other hand, "Claiming Space" by van der Meer takes the camera as an architectural legacy control tool to intervene and claim subversively the public space.
\end{abstract}

Keywords: REVEAL; REVOLT; ARCHITECTURE; VIDEO SURVEILLANCE; SPATIALITY

\footnotetext{
Pérez Suárez, Ventura Alejandro. 2017 . "El espacio perdido: Tácticas de contracultura en el arte (2016)". AusArt 5 (1): 179-191 D0I: 10.1387 /ausart.17460
}

\section{AUSART}




\section{1- INTRODUCCIÓN}

"I don't know why people are so keen to put the details of their private life in public; they forget that invisibility is a superpower"

(Banksy)

El término cultura hace referencia a una gran gama de significados y que se aborda, en una primera aproximación por Edward Tylor ([1871] 1981), como un sistema complejo compuesto por diferentes ámbitos de conocimiento entre los que podemos destacar cualquier campo que relacione al hombre con la sociedad (política, derecho, sociología, arte...).

Además, el investigador García Naharro (2012) posiciona la cultura como objeto de estudio antropológico social, el cual entiende la cultura como forma de vida y código de conducta. Esta definición que relaciona la cultura como un conjunto de normas en la conducta social genera una combinatoria de "microculturas" que se acerca o aleja a unos valores establecidos por una "macrocultura" que ha de establecerse bajo una condición de orden, vigilancia y control espacial.

Por consiguiente, se genera una subcultura si el código de valores difiere de modo pasivo al grupo dominante; mientras que la contracultura llega a distanciarse de la cultura central para cuestionar ese sistema que tiende a volverse totalitario (Roszak [1968] 1981).

De este modo se propone en este artículo la revisión de dos obras que plantean una nueva forma de conciencia crítica propia de la contracultura contemporánea y que inciden en el hecho de recuperar el espacio ganado por la cultura central. Por consiguiente, en estas obras se analiza una nueva dimensión a un orden espacial y cultural imperante que, según advierte Didier Bigo, ya no utiliza los métodos físicos arquitectónicos del panóptismo tanto como el banóptismo ${ }^{1}$ y su videovigilancia (Bauman \& Lyon 2013). 


\section{2- TEORÍA DE LA APROPIACIÓN INVERSA}

"La Cultura Popular, la Cultura Popular y los Mass Media convierten a Beethoven en algo fácil de silbar".

(Cultura popular - Siniestro Total)

Los espacios fotografiados en el proyecto de Paula Artés (1996, Molins de Rei) nos revelan aquellos lugares de trabajo marcados por la aparente ausencia de sus operantes; la Guardia Civil en Cataluña.

Oficinas, salas de monitores conectados a una red de videovigilancia, pasillos y diferentes útiles aportan claridad sobre el desconocimiento por parte del espectador del espacio que ocupa la seguridad. En consecuencia, este proyecto debe su nombre a la ley que nombra las competencias de la Guardia Civil catalana; L.2/86.

El interés de la obra "L.2/86" parte de la cuestión foucaultiana de los roles entre vigilante y vigilado; cuando este primero hace suyas las dinámicas de ocultación que se le atribuyen al segundo. Como se suele creer, la artista explica que el poder se asocia habitualmente con la captación de visibilidad cuando, en realidad, el poder actual también toma sus ventajas en su propia ocultación.

En definitiva, "L.2/86" refuerza su sentido al posicionarse más allá de los propios bastidores donde la vigilancia se hace invisible y por lo tanto efectiva, haciendo explícito su mecanismo velado.

Es destacable como "L.2/86" se posiciona en una crítica contracultural sin ejercer ningún tipo de violencia contra la integridad de la videvigilancia camuflada. Por lo tanto, se incide en que el posicionamiento de esta obra es contracultural en cuanto revela y crítica un modo de vigilancia camuflado pero jugando desde las normas impuestas por el mismo. En ningún momento esta obra pasa a infringir ninguna ley, ya que llega infiltrarse en su aparato legal mediante un órgano burocrático de autorización para poder fotografiar estos espacios. Después de un año de espera, la artista obtuvo los permisos para empezar a estudiar los espacios que descubriría bajo la supervisión de un cuerpo de la Guardia Civil. 
Artés concluye con un proyecto en el que no hace falta salirse del sistema para criticar al sistema. ¿Está bien evidenciar lo oculto? ¿Se podría entender este proyecto como publicidad positiva al órgano de vigilancia?
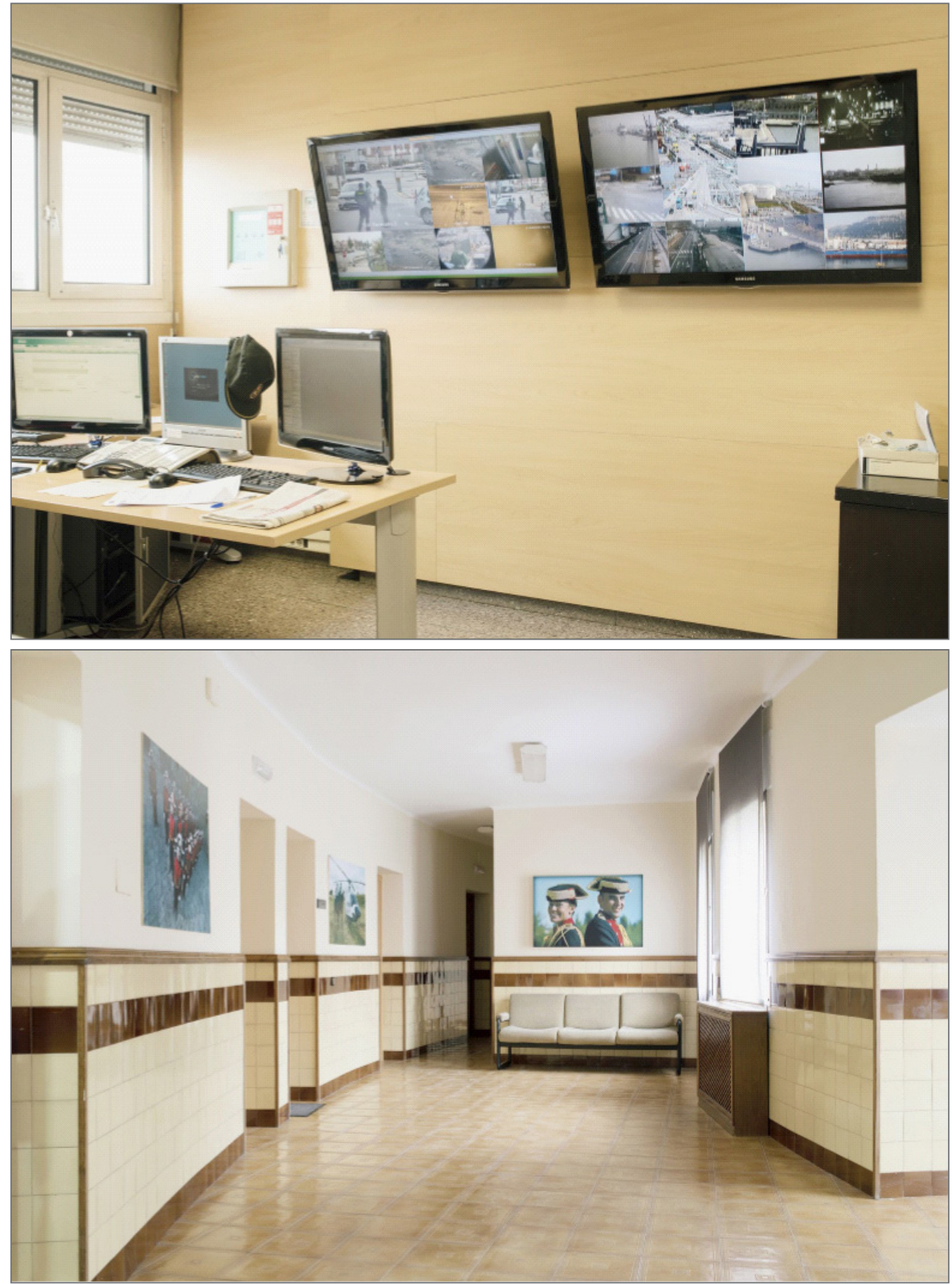


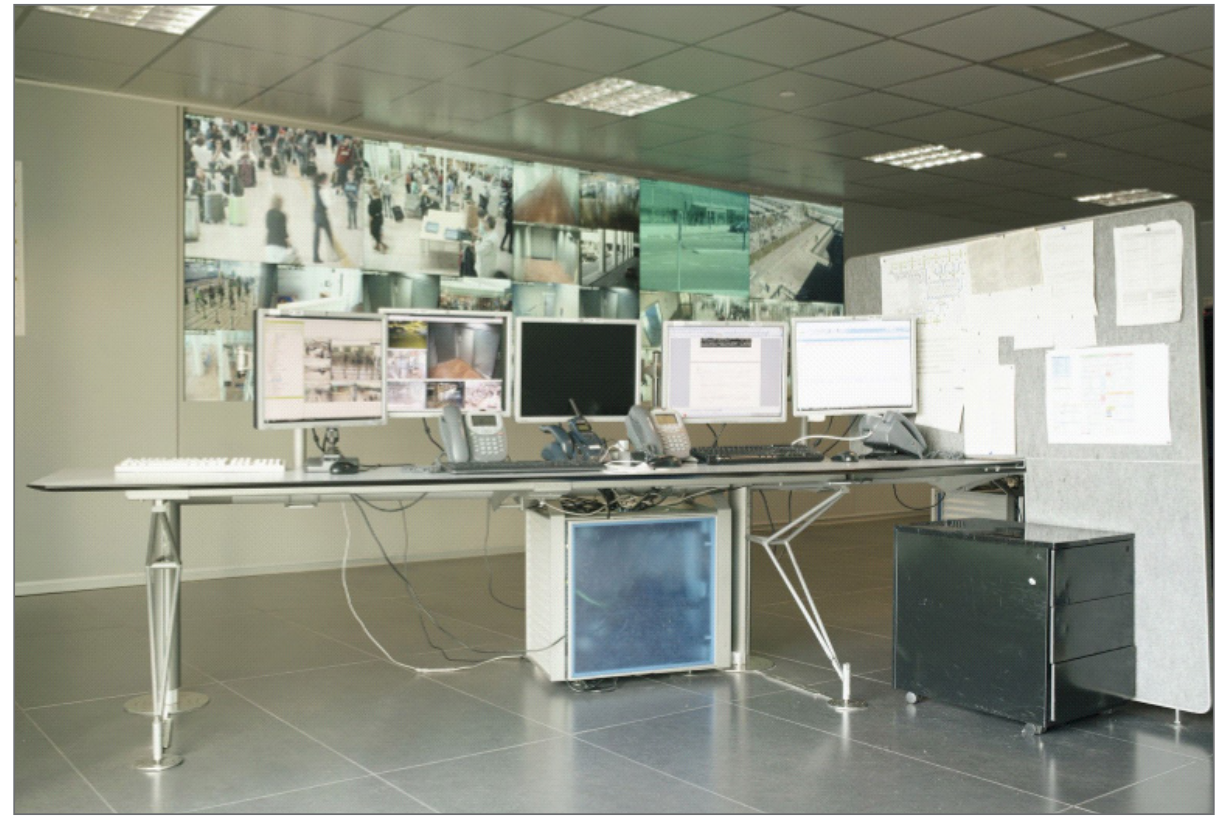

Fig. 1, 2 y 3. Paula Artés, L.2/86. 2016. Fotografía.

En este caso, las respuestas son tanto afirmativas como negativas, y ahí se demuestra este posicionamiento de indefinición. La artista había contado finalmente con la colaboración y apoyo de la Guardia Civil ya que el valor propagandístico de estas fotografías también atestiguan la función que cumplen dichos trabajadores minusvalorados por su invisibilidad. Si la crítica parece ser dignificadora, más bien es todo lo contrario.

Desde una perspectiva más amplia, y como ha demostrado la obra de Artés, los mecanismos de la cultura central pasan a intercambiar sus cualidades de visibilización por invisibilización. Lo que antes se creía como cultura central y visibilizada pasa a ser una tecnocracia equipada "con técnicas de manipulación de la intimidad tan finas y discretas como una telaraña" (Roszak [1968] 1981). Detrás de esta manipulación está la idea de que en la ciudad contemporánea "ya no puede existir lo oculto y desconocido, que ya no habrá sitio donde esconderse ni donde esconder nada" (García Cortés 2010), mientras que la subversión de la contracultura pasa a ser exhibida por una supracultura de "tolerancia represiva" descrita por Marcuse (citado en Heath \& Potter 2005), donde el bloqueo se produce a través de la asimilación, condescendencia y discriminación positiva por parte de la supracultura. 
Del mismo modo, es lícito pensar que para criticar esta nueva cultura central, sutil, camuflada y que exige transparencia al resto, se debe aceptar este intercambio de papeles y tomar sus antiguas tácticas de tolerancia represiva contra ella. Una de estas pasadas prácticas que había tomado la antigua cultura central en detrimento de la contracultura era el proceso de la publicidad y que Artés subvierte a su favor.

Roszak en el "Nacimiento de una Contracultura" (1969) ya advertiría el poder de los media a la hora de estereotipar y deshacer la contracultura americana gestada a partir de los 50 y madurada en los 60 con el movimiento hippie y beatnik.

Por aquel entonces, tanto el marketing, el cine americano o los expertos técnicos de la tecnocracia (sociólogos, psicólogos, etc.) neutralizarían estas contraculturas caracterizadas por la disconformidad y crítica contra el totalitarismo de un sistema y cultura hegemónicos, mediante la infiltración y asimilación de sus conductas rebeldes. "Una especie de cínica eliminación de la protesta por el manto de saturación que la lanza la sociedad" (Roszak 1969), donde los infiltrados ("hippies domingueros" o la "moda punk"), boicotearían la contracultura en subcultura mediante el estereotipado y vulgarización para, posteriormente, transformar esta subcultura en un añadido del gran aparato central cultural.

Como conclusión en su época, la contracultura aprendería a que es necesario distanciarse y tomar la subversión, ocultamiento y recelo como formas de supervivencia (Roszak 1969).

Otros autores como Heath y Potter volverían a retomar en la actualidad, bajo su investigación "Rebelarse vende" (2004), el proceso de absorción de subculturas y contraculturas bajo la teoría de la apropiación, cuya premisa reside en vaciar de contenido revolucionario de la contracultura y vender el envoltorio vacío restante.

Sin ir más lejos, España contó con el caso de la movida madrileña y viguesa como un movimiento subcultural. Este fue absorbido por la publicidad y comercialización de una política socialista de los 80 que buscaba una nueva imagen. Desde entonces, el marketing de la movida se convirtió en aquel dispositivo a favor de una nueva visión a la España de la Transición (García Naharro 2012).

Pero se debe resaltar que la movida se estableció como subcultura y no como contracultura, ya que no perseguían un ideal crítico contra lo establecido tanto 
como el pasotismo, despolitización y moral consumista (García Naharro 2012). La absorción de la movida a la gran estructura cultural no fue una pérdida de un valor crítico contracultural aunque resulte un ejemplo interesante en la marketización a la española de aquel entonces.

Volviendo a la obra de Artés, la dinámica de la apropiación reaparece en su obra al ofrecer visibilidad y glorificación a la Guardia Civil por medio de documentar un despliegue técnico de videovigilancia cuando, por otro lado, denuncia al mismo su práctica invisibilizada.

Se trata de responder consecuentemente ante un cambio de roles paradójico; donde extrañamente la contracultura ofrece una cara amable, moviendo su crítica bajo el marco legal y del media mientras que por otro lado, la Guardia Civil encuentra un reflejo o reconocimiento en la obra de Artés ante unas tácticas de trabajo que exigen una ocultación muchas veces desprestigiada por los ciudadanos.

En definitiva, "L.2/86" aporta una teoría de apropiación a la inversa: ofrecer el contenido crítico en un envoltorio amable, dignificador o inocuo.

\section{3- LO ARQUITECTÓNICO SE DESVANECE EN LA CÁMARA}

Como se ha visto en el apartado anterior, la videovigilancia en el medio urbano supone un doble filo para el vigilante y el vigilado. Quien vigila ha de situarse fuera de un marco social y cultural que lo apoye, siendo a veces su trabajo estigmatizado. Es ahí donde Artés concluye con una obra que radica su eficacia en una crítica camuflada.

Mientras tanto, otros procedimientos se establecen con el propio objeto que media los roles de vigilante y vigilado; la cámara. Si la obra de Artés aborda una dialéctica vertical entre la Guardia Civil y el ciudadano. ¿Qué ocurre si se incluye la cámara de video en una estructura de diálogo horizontal entre ciudadanos? 
El texto de un supuesto viandante en la página web de Leendert van der Meer (Veenendaal , 1992) nos dice: "Mientras caminaba por Binnenrotteplein, mi dirección era corregida y mi ruta interrumpida" - y continúa- "La presencia de turistas fotografiando formaba un obstáculo. Un simple acto como caminar se volvía complejo. Aunque normalmente cruzo la plaza en diagonal, esta vez tuve que zigzaguear" (Van der Meer 2016).

La propuesta de "Claiming Space" hace honor a su nombre al reclamar el espacio público por medio de la cámara fotográfica. Esta última se relee como herramienta de poder y creadora de nuevas espacialidades cuando Van der Meer nos muestra una intervención en el espacio público.

En esta obra, cuatro de sus compañeros con sus cámaras y trípodes se posicionan en una de las plazas más famosas de Rotterdam. Su emplazamiento se conforma a partir de un cuadrado imaginario en el suelo, en cuyos vértices se colocan los fotógrafos, formando un espacio imposible de atravesar sin haber sido interceptado por las cámaras dirigidas al interior de esta forma imaginaria. Un quinto operante, Leendert, filma lo ocurrido en Binnenrotteplein desde las alturas de un edificio.

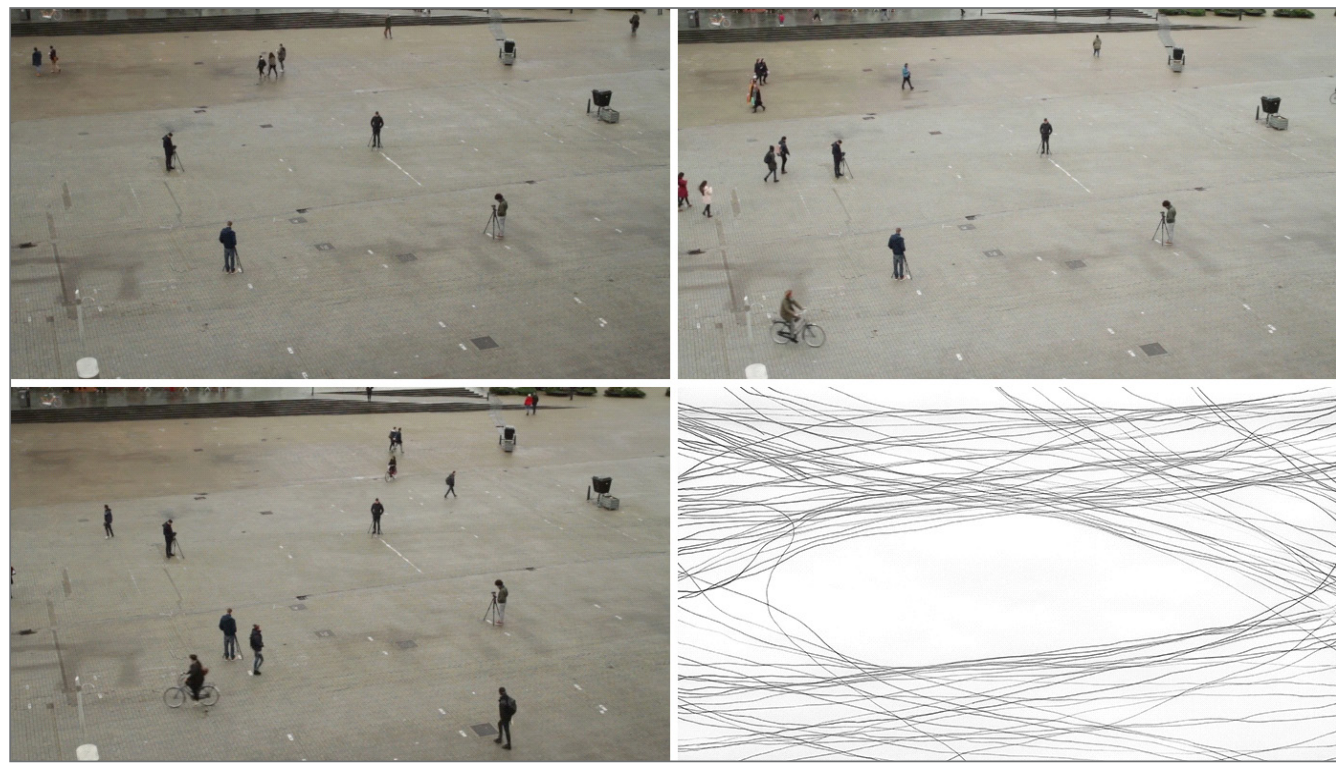

Figura 4, 5, 6 y 7. Leendert van der Meer, Claiming space. 2015-2016. Intervención, vídeo y papel trazado. 
Finalmente, el artista recopila y calca con líneas en un papel los diferentes recorridos de las personas filmadas. Un gran espacio en blanco aparece en medio de este dibujo y coincide con el espacio intervenido en la plaza, demostrando que ninguno de los transeúntes se atrevieron a transgredir los límites imaginarios y establecidos por la obra. Una nueva especie de muro se había levantado en mitad de grandes arquitecturas como el Markthal o la Blaaktoren.

¿Se debería tratar esta intervención como un nuevo tipo de arquitectura? Desde sus comienzos, la arquitectura ha desarrollado su función en base a contenidos duales (interior y exterior, público y privado, estabilidad y cambio...) bajo la dirección de una cultura central, esta última ya anteriormente abordada en base a un código de conducta social.

Por lo tanto, la arquitectura que se posiciona a favor de este tipo de modelo cultural-social se transforma en una de las representaciones de poder más efectivas a la hora de corroborar la centralidad y supremacía de la supracultura (García Cortés 2010).

Esto es debido a que, como apuntan Heath y Potter, "es frecuente que no reparemos en los rasgos más sobresalientes de nuestro entorno precisamente por lo ubicuos que son" (Heath \& Potter 2005). La arquitectura es ubicua en tanto que conforma el entorno urbano en el que vivimos, y el entorno se da por sentado o se vuelve implícito sin una mirada crítica adecuada. De este modo, esta disciplina espacial siempre se hace a un segundo plano si se considera un mero telón de fondo cuando, en realidad, intercepta las acciones y conductas de sus habitantes.

La gran ventaja que ha tenido la cultura central al acceso de poder es debido a la ayuda de la arquitectura, que "posee la habilidad para esconder sus propios mecanismos y consigue hacer pasar por bien general aquello que no es más que legitimación de su poder" (García Cortés 2010). Pero diferentes contraculturas han desafiado esta monopolización espacial. Esto se hace notar en el choque entre estos dos tipos de culturas y que da como resultado una ciudad bicéfala.

La primera de estas caras urbanas impera bajo el dominio de la cultura central y se compagina en base a una construcción de espacios normalizados. Es el caso de la arquitectura del centro comercial para comprar la nueva colección "grunge" o "punk" de la temporada, la arquitectura del cine para ver la última 
película comercial, la arquitectura del supermercado que un actor famoso patrocina, etc.

La segunda cara nos ofrece una nueva lectura impuesta por diferentes tipos de contraculturas, que aunque normalmente no trabajen desde sus construcciones propias, reutilizan sin dejar rastro el espacio arquitectónico y urbano en base a otros propósitos. El fenómeno de "Post-it City" los definirá como la respuesta a una presión homogeneizadora del espacio público por parte de la cultura de masas. Se trata, en definitiva, de la intervención "frente a los ideales de la ciudad como lugar de consenso y de consumo, (...), desvelan distintas necesidades y carencias que afectan a determinados colectivos, e incluso potencian la creatividad y el imaginario subjetivo" (Post-it City 2013).

El ejemplo del cruising en baños públicos o parques, movimientos okupas en edificios abandonados o campamentos ilegales son algunos de los casos de diferentes colectivos que reclaman un espacio para ellos a la hora de reutilizar los ya dados. Además, este solapamiento de funciones en un mismo espacio genera una cultura en si misma en contra de la norma. "La regla es simple: cuantos más territorios superpuestos haya en una zona determinada, más circulación habrá entre ellos, y menos influencia tendrá el poder" (Comité invisible, 2009).

Sennett, en "Carne y Piedra" (1994) insiste en el hecho de que el diseño urbanístico a lo largo de la historia genera un comportamiento y conducta a favor de una cultura y grupo social privilegiados. De hecho, muestra el ejemplo de la Revolución francesa como fenómeno contracultural que es inhibido por la transformación urbanística de París. Este bloqueo se consiguió con el abatimiento de los muros y rincones de la capital, donde no habría posibilidad para la congregación y escondite de los sublevados (Sennett [1994] 2015). París transformaría sus calles en espacios despejados para una mejor vigilancia.

Consecuente a esas aperturas, Foucault examina una ciudad contemporánea bajo el prisma social disciplinario, donde el sólido bloqueo del muro o la puerta cerrada pasa a ser sustituido "por el cálculo de las aberturas, de los plenos y de los vacíos, de los pasos y las transparencias" (Foucault [1975] 1998).

Lo sólido arquitectónico se derrite a una transparencia propia de la arquitectura de Mies van der Rohe, para evaporarse en el culmen de las CCTV (cámaras de vigilancia adosadas a edificios) como el último legado de las tácticas 
espaciales de control normativo y que Leendert toma a su favor de manera subversiva.

"Claiming space" no se construye a través de la propia arquitectura al uso, tampoco la reutiliza como en el caso abordado del "Post-it city". Demuestra otra alternativa al reclamar un espacio fuera del marco legal constructivo y al mismo modo de apropiación que Santiago Cirugeda o SpY generan en su línea de trabajo. Esta intervención generada, como se ha visto también en la obra de Paula Artés, subvierte a su favor las tácticas escópicas y arquitectónicas de la cultura central para ir en su contra.

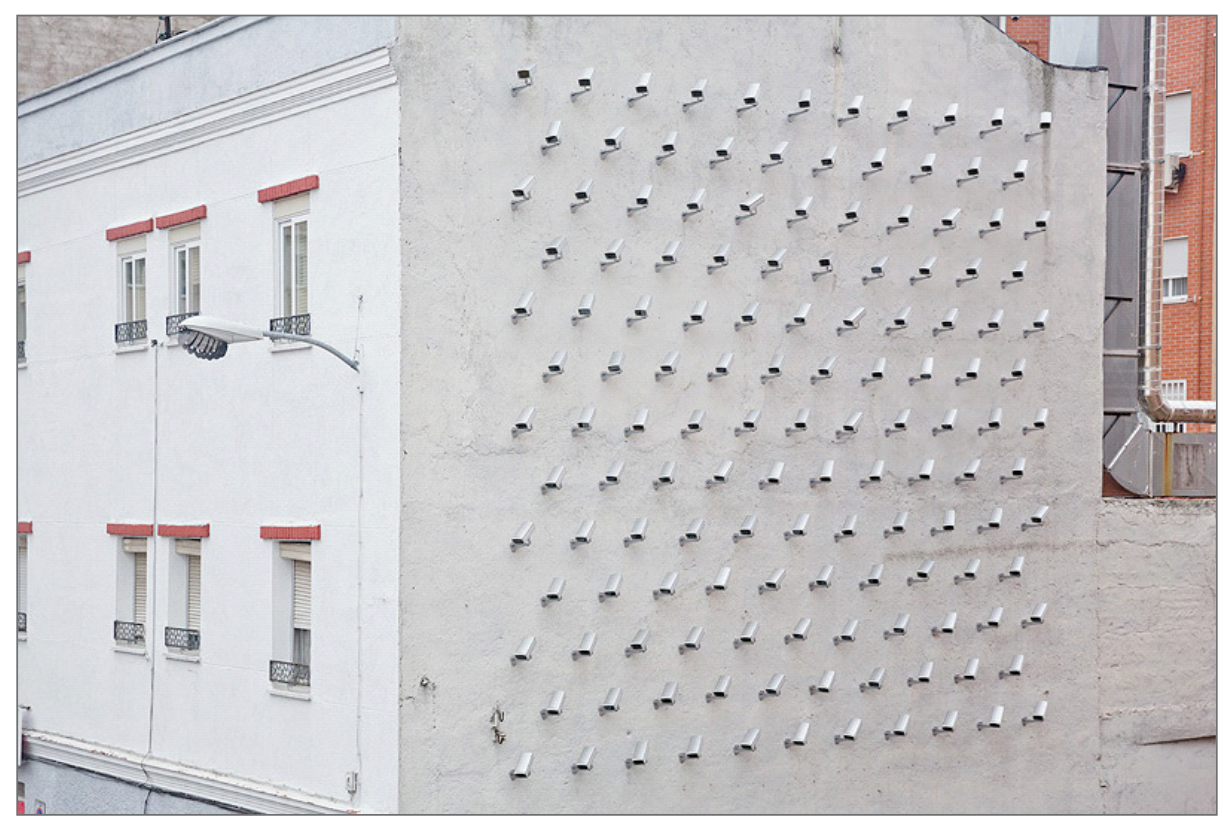

Figura 8. SpY, Cameras. 2013. Instalación.

\section{4- CONCLUSIÓN}

El trabajo abordado de estos dos artistas muestran algunas de las posiciones contraculturales en el arte contemporáneo. A su vez, la constante que los acerca es el del análisis del espacio público y sus maneras de reclamar la parte privada y perdida en este. 
El despliegue técnico de la Guardia Civil fotografiado por Artés nos muestra un doble enfoque que desvela, por un lado, el gran mecanismo de control actual mientras que, por otro lado, induce a la Guardia Civil a un mismo grado de visibilización. Por consiguiente, "L.2/86" genera una pregunta: ¿Quién ha de vigilar a los que vigilan? En esta cuestión y en el propio visionado de la obra de Artés se incide en que aún sigue existiendo un espacio oculto fuera de todo el despliegue técnico de control y que es aquel en donde se instala ese mismo ejercicio de vigilancia.

De otro modo, una visión más positiva ante la pregunta que se cuestiona por un espacio subversivo es la ofrecida en "Claming Space". Leendert usará la cámara no como aquel objeto que media la verticalidad entre vigilante y vigilado, sino que estudia y experimenta el efecto de la cámara dentro de una estructura donde prime la horizontalidad de sus integrantes. Usando el espacio público y la dinámica de su circulación, la cámara parece generar barreras y direcciones para los transeúntes. Sin que el artista sea una autoridad dentro de las estructuras de control, "Claming Space" genera un nuevo espacio como diferentes subculturas y contraculturas han buscado y reutilizado para sus deseos y necesidades. Cabe destacar que la obra de van der Meer incide en que el acto de ver y vigilar no es solamente propio del poder de una cultura central.

En definitiva, los métodos y resultados son diferentes. Mientras que Paula Artés cuestiona la posición del vigilante y su camuflaje al generar un discurso crítico del "revelar para criticar" bajo otra apariencia más amable del "revelar para dignificar", Leendert van der Meer apuesta por una intervención donde la visibilidad genera barreras invisibles. La cámara es, en este último caso, la herramienta para visibilizar y, por lo tanto, de generar un contrapoder, que se apropia del espacio público.

Finalmente, el cuestionamiento del papel entre vigilante-vigilado (Paula Artés) o el reciclaje del medio a su favor (Leendert van der Meer) son algunas de las propuestas que tiene el arte como modelo contracultural y crítico a la hora de responder a la siguiente pregunta. ¿Existe otro espacio fuera de un sistema cultural invasivo y de vigilancia? 


\section{Referencias}

Comité invisible (Grupo). 2009. La insurrección que viene. Traducción de Yaiza Nerea Pichel Montoya. Barcelona: Melusina

Foucault, Michel (1975) 1998. Vigilar y castigar. Nacimiento de la prisión. Traducción de Aurelio Garzón del Camino. Madrid: Siglo XXI

García Cortés, José Miguel. 2010. La ciudad cautiva. Control y vigilancia en el espacio urbano. Madrid: Akal

García Naharro, Fernando. 2012. "Cultura, subcultura, contracultura: 'Movida' y cambio social (1975-1985)". En Coetánea: III Congreso Internacional de Historia de Nuestro Tiempo, Carlos Navajas Zubeldía, Diego Iturriaga Barco, coords., 301-10. Logroño: Univ. de La Rioja. https://dialnet.unirioja.es/servlet/articulo?codigo $=4052246$

Heath, Joseph \& Andrew Potter. 2005. Rebelarse vende. El negocio de la contracultura. Traducción de Gabriela Bustelo. Madrid: Taurus

Post-it City. 2013. Post-it City. Ciudades ocasionales, blog del proyecto. Anna Recasens, coord. Acceso 6 diciembre. http://www.ciutatsocasionals.net/homepage.htm

Roszak, Theodore (1968) 1981. El nacimiento de una Contracultura: Reflexiones sobre la sociedad tecnocrática y su oposición juvenil. Traducción por Angel Abad. Barcelona: Kairós

Sennett, Richard (1994) 2007. Carne y Piedra: El cuerpo y la ciudad en la civilización occidental. Traducido por César Vidal. Madrid: Alianza

Tylor, Edward (1871) 1981 Cultura primitiva. Traducción por Marcial Suárez. Madrid: Ayuso

Van der Meer, Leendert. 2016. "Claiming space". Leendertvandermeer.com [web personal del autor]. Acceso 6 diciembre. http://www.leendertvandermeer.com/claiming.html

Zygmunt, Bauman \& David Lyon. 2013. Vigilancia líquida. Traducción de Alicia Capel Tatjer Barcelona: Paidós

\section{Notas}

${ }^{1}$ Banóptismo es el término emplado por diferentes teóricos como Didier Bigo o Zygmunt Bauman para señalar un modo de vigilancia informatizada que ya no se centra en el clásico control del encierro, tratado en las arquitecturas carcelarias panópticas de Bentham o desde la postura teórica de Foucault, sino que opera bajo el acto de exclusión, expulsión o alejamiento de zonas bajo videovigilancia. El término Banópico viene del inglés "ban": exclusión. En definitiva se trata de una "vigilancia de exclusión". 